\title{
Correlation of Treatment Compliance, Treatment Attitude and Belief, and Quality of Life in Community Hypertensive Patients
}

\author{
Xiao-Jiao LIU ${ }^{1}$, Hong-Fang CHEN ${ }^{2 *}$, Jia-Mei LI², Yuan LYU², Sheng-Rui ZHU ${ }^{3}$, Huan-Ze LI ${ }^{1}$ \\ ${ }^{1}$ Shaanxi University of Chinese Medicine, Xianyang, China \\ ${ }^{2}$ Shaanxi Traditional Chinese Medicine Hospital, Xi'an, Shaanxi, China \\ ${ }^{3} X i$ 'an Jiaotong University City College, Xi'an, Shaanxi, China
}

\begin{abstract}
Objective: To explore the relationship between treatment compliance, treatment attitude and belief, and quality of life in patients with hypertension in the community, and to provide evidence for improving their quality of life.

Methods: A convenient sampling method was used to survey 250 patients with hypertension who met the inclusion criteria using the General Questionnaire, Treatment compliance, Treatment Attitudes and Beliefs, and Quality of Life Scale.

Results: The total score of treatment compliance of hypertension patients in the community was (91.678 \pm 11.431$)$,

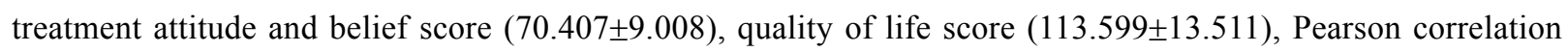
analysis showed that the treatment compliance of hypertension patients was positively correlated with quality of life $(r=0.433, P<0.01)$, and treatment attitude and belief were positively correlated with quality of life ( $r=0.463$, $P<0.01)$.
\end{abstract}

Conclusion: The quality of life of patients with hypertension in the community is related to treatment compliance and treatment attitudes and beliefs.

Key words: Hypertension; Treatment compliance; Treatment attitudes and beliefs; Quality of life

\section{Introduction}

Hypertension is a chronic non-communicable disease that threatens the health of the world and the health of our country. It is an irreversible life-long disease and one of the key diseases in community chronic disease management. With the development of social economy, the increase of life pressure, lifestyle changes, etc., the incidence of hypertension continues to increase. The

\footnotetext{
*Corresponding Author:

Hong-Fang CHEN, female, master's degree, deputy director of the nurse, nursing management, Shaanxi Traditional Chinese Medicine Hospital, Xi'an 710003, China E-mail: 13928881695@163.com

Received: October 6 2019, Revised: October 16 2019, Accepted: October 202019 , Online: November 152019
}

management of hypertension is a long-term process. If you can not effectively control blood pressure, it will eventually lead to important organ complications such as heart, brain and kidney, which will seriously affect the life and quality of life of patients ${ }^{[1]}$. Some studies have suggested that ${ }^{[2]}$, patient treatment compliance is an important prerequisite for ensuring treatment effect, reducing complications and improving patients' quality of life. In recent years, researchers have gradually introduced social psychology factors to improve the therapeutic compliance of patients with hypertension ${ }^{[3]}$. 
Studies have shown that patients' attitudes and beliefs about anti hypertensive therapy are the influencing factors of treatment compliance and treatment effect ${ }^{[4]}$. Therefore, this study enrolled hypertension patients in two communities in Shanxi Province as the survey object to explore the relationship between treatment compliance, treatment attitude and belief, and quality of life. It provides reference for improving patients' treatment compliance and improving their quality of life. The report is as follows:

\section{Research objects and methods}

\section{Research object}

The survey respondents were eligible for hypertension in two communities in Shanxi Province from April 2019 to June 2019. Inclusion criteria were that patients must meet the following conditions: (1) the diagnostic criteria for hypertension ${ }^{[5]}$, (2) age 18 75 years, (2) living in the survey area $\geq 1$ year, (4) no mental and cognitive impairment or no such signs presently, (4) understanding their condition, volunteering to participate in this study. Exclusion criteria were that patients had serious organ diseases such as severe heart, kidney, liver failure or patients had mental illness.

\section{Survey method}

The researchers distributed questionnaires to 250 patients who met the inclusion criteria, explained the purpose and precautions of the survey to the patient in a unified instruction, and answered the questions from the patients. The questionnaires were completed independently and collected on the spot. 236 effective questionnaires was recovered, and the effective recovery rate was $94.4 \%$. All the patients signed informed consents before the completion of questionnaire.

\section{Research Tools}

General Information Table. It includes demographic characteristics and history of illness and family history.

Therapeutic compliance Scale. This scale was compiled by Tang Hongying ${ }^{[6]}$, including four dimensions of compliance with medication behavior, poor medication behavior, daily life management behavior, and tobacco and alcohol hobby management behavior. This scale contains 25 entries in total, The Cronbach's $\alpha$ of the scale is 0.863 .

Therapeutic Attitude and Belief Scale. This scale was compiled by Tang Hongying ${ }^{[7]}$, including medication treatment attitudes and beliefs, treatment of lifestyle attitudes and beliefs, persistent treatment distress concerns, adherence to treatment attitudes and beliefs, and a total of 21 items. The Cronbach's $\alpha$ of the scale is 0.804 .

Quality of Life Scale (SF-36). Developed by the Boston Institute of Health in the United States, it has been widely used to measure the quality of life of the general population $^{[8]}$, and can comprehensively assess the physical, psychological and social functions of individuals and groups. Divided into two aspects of physical health and mental health, including physiological functions, physiological functions, physical pain, general health, social function, emotional function, mental health, vitality and health changes, a total of 36 items. According to the content of each dimension, the problem entries are set, each item is coded, the scores of each item are added to the actual score of the dimension, and then the actual score is converted into a standard score according to the formula, and the sum of the scores of each dimension is SF-36. Standard score $=$ (actual score - lowest score for this dimension) / (highest score for this dimension-lowest score for this dimension) $\times 100^{[9]}$.

\section{Statistical analysis}

Statistical analysis was performed using SPSS19.0. The measurement data were presented as mean \pm standard deviation $(\bar{x} \pm s)$, the frequency and composition ratio of the count data were used, and the differences between groups were compared by analysis of variance and test. The correlation between variables was analyzed by Pearson correlation analysis.

\section{Results}

General information includes demographic characteristics and history of illness and family history, using frequency 
and composition ratio for statistical description (Table 1). The compliance of treatment on hypertension patients was shown in Table 2. The treatment attitude and belief scores of hypertensive patients were shown in Table 3. The quality of life score of patients with primary liver cancer was shown in Table 4. The correlation between treatment compliance, treatment attitude and belief, and quality of life using Pearson correlation analysis is shown in Table 5.

Table 1 General information of patients

\begin{tabular}{|c|c|c|}
\hline Variables & Number of cases & Composition ratio \\
\hline \multicolumn{3}{|l|}{ Age (years) } \\
\hline $18-44$ & 14 & $5.9 \%$ \\
\hline $45-59$ & 142 & $60.1 \%$ \\
\hline $60-75$ & 80 & $34.0 \%$ \\
\hline \multicolumn{3}{|l|}{ Gender } \\
\hline Male & 101 & $42.8 \%$ \\
\hline Female & 135 & $57.2 \%$ \\
\hline \multicolumn{3}{|l|}{ Educational level } \\
\hline Primary school & 20 & $8.5 \%$ \\
\hline Middle school & 151 & $64.0 \%$ \\
\hline Undergraduate & 65 & $27.5 \%$ \\
\hline \multicolumn{3}{|l|}{ Marriage } \\
\hline Married & 178 & $75.4 \%$ \\
\hline Unmarried/divorced/ widowed & 58 & $24.6 \%$ \\
\hline \multicolumn{3}{|l|}{ Career } \\
\hline In-service & 62 & $26.3 \%$ \\
\hline Retirement & 40 & $16.9 \%$ \\
\hline Unemployed & 134 & $56.8 \%$ \\
\hline \multicolumn{3}{|l|}{ Income (RMB) } \\
\hline$\leq 2000$ & 17 & $7.2 \%$ \\
\hline 2000-3999 & 105 & $44.5 \%$ \\
\hline $4000-5999$ & 98 & $41.5 \%$ \\
\hline$\geq 6000$ & 16 & $6.8 \%$ \\
\hline \multicolumn{3}{|l|}{ Type of medical insurance } \\
\hline Medical insurance & 168 & $71.2 \%$ \\
\hline NCMS & 32 & $13.6 \%$ \\
\hline Self-pay & 36 & $15.2 \%$ \\
\hline \multicolumn{3}{|l|}{ Hypertension grading } \\
\hline Level 1 & 133 & $56.3 \%$ \\
\hline Level 2 & 57 & $24.2 \%$ \\
\hline Level 3 & 46 & $19.5 \%$ \\
\hline \multicolumn{3}{|l|}{ Cardiovascular family history } \\
\hline Have & 51 & $78.4 \%$ \\
\hline No & 185 & $21.6 \%$ \\
\hline \multicolumn{3}{|l|}{ Complication } \\
\hline Have & 12 & $5.1 \%$ \\
\hline No & 224 & $94.9 \%$ \\
\hline
\end{tabular}


Table 2 Treatment compliance scores of hypertensive patients

\begin{tabular}{lcccc}
\hline Items & $\begin{array}{c}\text { Maximum } \\
\text { (Minute) }\end{array}$ & $\begin{array}{c}\text { Minimum } \\
\text { (Minute) }\end{array}$ & Score $(\bar{x} \pm s)$ & Scoring rate (\%) \\
\hline Medication Compliance & 5.00 & 25.00 & $18.754 \pm 4.186$ & 75.016 \\
Poor medication compliance & 8.00 & 40.00 & $30.004 \pm 6.035$ & 75.010 \\
Life management compliance & 20.00 & 50.00 & $35.030 \pm 4.740$ & 70.060 \\
$\begin{array}{l}\text { Tobacco and alcohol management } \\
\text { compliance }\end{array}$ & 2.00 & 10.00 & $7.890 \pm 2.234$ & 78.901 \\
Dependency total score & 54.00 & 125.00 & $91.678 \pm 11.431$ & 73.342 \\
\hline
\end{tabular}

Note: Scoring rate=actual score of each item / theoretical maximum score $\times 100 \%$

Table 3 Treatment attitude and belief scores of hypertensive patients

\begin{tabular}{lcccc}
\hline Items & $\begin{array}{c}\text { Maximum } \\
\text { (Minute) }\end{array}$ & $\begin{array}{c}\text { Minimum } \\
\text { (Minute) }\end{array}$ & Score $(\bar{x} \pm s)$ & Scoring rate (\%) \\
\hline Medication belief & 7.00 & 35.00 & $21.754 \pm 4.453$ & 62.154 \\
Lifestyle belief & 15.00 & 35.00 & $23.746 \pm 3.707$ & 67.845 \\
Treatment confusion & 4.00 & 13.00 & $9.305 \pm 1.718$ & 62.033 \\
Adhere to the belief in treatment & 10.00 & 20.00 & $15.602 \pm 2.691$ & 78.010 \\
Total score of attitude and belief & 49.00 & 102.00 & $70.407 \pm 9.008$ & 67.054 \\
\hline
\end{tabular}

Note: Scoring rate=actual score of each item / theoretical maximum score $\times 100 \%$

Table 4 Quality of life scores of hypertensive patients

\begin{tabular}{lcccc}
\hline Items & $\begin{array}{c}\text { Maximum } \\
\text { (Minute) }\end{array}$ & $\begin{array}{c}\text { Minimum } \\
\text { (Minute) }\end{array}$ & Score $(\bar{x} \pm s)$ & $\begin{array}{c}\text { Scoring rate } \\
\text { (\%) }\end{array}$ \\
\hline Physiological function & 14.00 & 30.00 & $27.076 \pm 3.218$ & 90.253 \\
Physiological limitations & 4.00 & 12.00 & $6.976 \pm 1.708$ & 87.200 \\
Somatic pain & 2.00 & 36.00 & $9.582 \pm 2.407$ & 87.109 \\
Overall health & 10.00 & 60.00 & $17.133 \pm 3.856$ & 68.532 \\
Physiological health score & 41.00 & 85.00 & $60.769 \pm 6.747$ & 82.120 \\
vitality & 7.00 & 24.00 & $17.255 \pm 3.300$ & 71.895 \\
Social function & 3.00 & 12.00 & $8.632 \pm 1.666$ & 86.320 \\
Emotional function & 3.00 & 26.00 & $5.632 \pm 2.117$ & 93.866 \\
Mental Health & 6.00 & 30.00 & $21.347 \pm 4.352$ & 71.156 \\
Mental health score & 27.00 & 82.00 & $52.840 \pm 8.669$ & 75.485 \\
Total quality of life & 82.00 & 149.00 & $113.599 \pm 13.511$ & 78.888 \\
\hline
\end{tabular}

Note: Scoring rate=actual score of each item / theoretical maximum score $\times 100 \%$ 


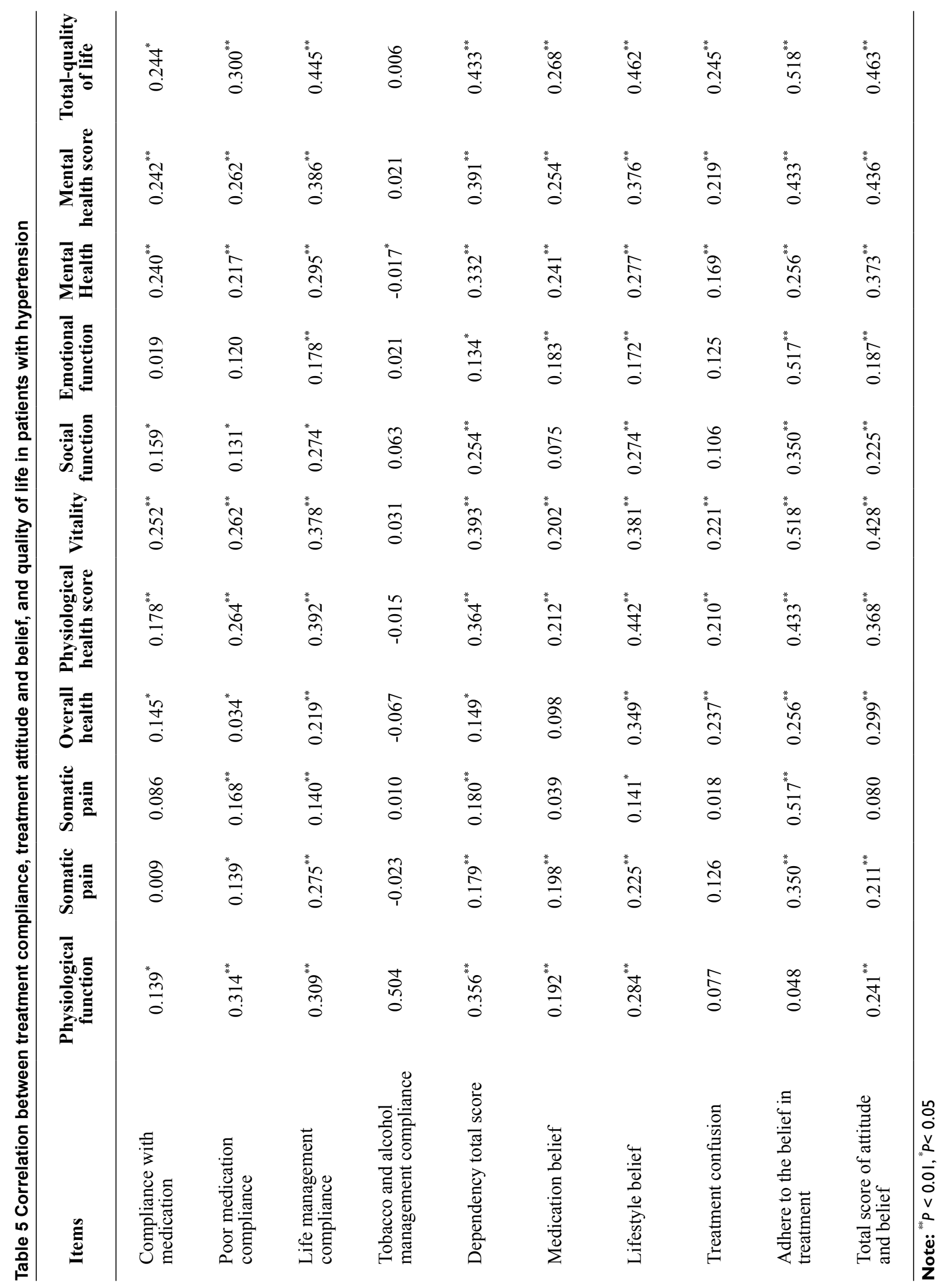




\section{Discussion}

The quality of life of patients with hypertension in the community is at a medium level. The results of the study showed that the patient's quality of life score was (113.599 \pm 13.511 points, See Table 4) and the score was $78.888 \%$, which was at a medium level, which was consistent with the results of Kitaoka ${ }^{[10]}$ and $\mathrm{Xu}$ et $a l .{ }^{[11]}$. In the sub-scales of patients' quality of life, the physiological health score rate is higher than the mental health score rate, indicating that the quality of life of patients with hypertension in the community is mainly mental health status. This may be due to hypertension as a chronic, lifelong disease, poor long-term blood pressure control, disease risk factors and complications need to be frequently treated and the treatment effect is not good, which brings a greater psychological burden to patients ${ }^{[12]}$. Therefore, community medical workers should promptly identify the psychological changes of patients, pay attention to mental health, and promote the improvement of the disease. At the same time, it is hoped that community medical workers will pay attention to the health education work of community residents, especially hypertensive patients, and help the community to cope with hypertension patients. The physical and psychological damage caused by the disease.

There is a positive correlation between treatment compliance and quality of life in community hypertension patients. The results of this study shows that the patient's treatment compliance score is $(91.678 \pm 11.431$ points, See Table 2) which is at a medium level, is consistent with the results of Li et al. ${ }^{[13]}$. The result also shows that the patient's treatment compliance is positively correlated with quality of life $(P<0.01$, See Table 5$)$, that is, the treatment compliance of hypertensive patients has a positive predictive effect on quality of life, suggesting that good therapeutic compliance is beneficial to reduce the occurrence of complications and improve the quality of life of patients, and vice versa. Correlation analysis also shows that patients' life management compliance is more closely related to quality of life, indicating that life management of hypertensive patients is particularly important to improve their quality of life. Therefore, it is suggested that medical personnel should join the family in all-round and multi-level efforts to improve the living environment of patients, improve bad living habits, and practice good and healthy living habits throughout their life, and consciously form healthy behaviors. Furthermore, by regularly holding high-pressure knowledge lectures, video lectures, one-on-one health consultations, personalized health education, etc., the channels and opportunities for patients to communicate with professional medical staff are increased, and patients are urged to improve their treatment compliance ${ }^{[14]}$. In order to better control blood pressure and reduce complications, the quality of life of patients is improved.

There is a positive correlation between treatment attitude and belief and quality of life in community hypertension patients. The results of this study shows that the patient's treatment attitude and belief score is $(70.407 \pm 9.008$ points, see Table 3$)$, which is at a moderately low level, is consistent with the results of Martha and Hill ${ }^{[15]}$. This result may be related to the cultural level and income of the subjects. It also shows that patients' treatment attitudes and beliefs were positively correlated with quality of life $(P<0.01$, See Table 5), that is, the treatment compliance of hypertensive patients has a positive predictive effect on quality of life, suggesting that positive treatment attitudes and beliefs have an important role in improving patients' quality of life. Some studies ${ }^{[16,17]}$ have concluded that, positive treatment attitudes and beliefs are conducive to the improvement of patient compliance, reduce the incidence of complications, and improve clinical outcomes. This suggests that community medical staff will continue to conduct health knowledge lectures, community clinics, and pressure control techniques to strengthen the health knowledge of hypertension in order to improve the management of chronic diseases in the community and Cognition. In particular, attention and support should be given to patients with low academic qualifications, low income, and low social support ${ }^{[18]}$. While carrying out health education, medical staff organize and conduct effective patient interactions to share experiences and exchanges among patients with better disease management, so as to encourage other patients to establish positive treatment 
attitudes and beliefs, enhance confidence in treating diseases, and be proactive.

\section{Summary}

In summary, the quality of life of patients with hypertension in the community needs to be improved, and the quality of life and treatment compliance, treatment attitude and belief are positively correlated. This suggests that community health workers should focus on cultivating patients' positive attitudes and beliefs, the patient's life management compliance, lifestyle and adherence to treatment beliefs, etc. in the process of chronic disease management, thus improving their therapeutic compliance. At the same time, through regular health education lectures, community clinics and other means to strengthen the education of community hypertension patients, and to fully mobilize the patient's subjective initiative should be done, so that patients actively participate in the treatment and management of disease, in order to improve the clinical outcomes and the quality of life of patients.

\section{Declaration}

All authors of this article declare they have no conflicts of interest.

\section{Acknowledgements}

This study was approved by Shaanxi Provincial Key Research and Development Program (2017SF-294).

\section{References}

1 Zhang W. The effect of health education on the quality of life of patients with hypertension in the community. Guid Chin Med, 2019, 17(4): 2-3.

2 Paczkowska A, Kopciuch D, Nowakowska E, et al. Compliance Among Adolescents with Arterial Hypertension. Adv Clin Exp Med, 2016, 25(3): 441448.

3 Cuffee Y, Ogedegbe C, Williams NJ, et al. Psychosocial risk factors for hypertension: an update of the literature. Curr Hypertens Rep, 2014, 16(10): 1-11.
4 Tang YY, Zhong MM. Research progress on quality of life in patients with essential hypertension in Zhongqing. Nurs Res Chin, 2019, 33(13): 2276-2280.

5 Liu LS. Chinese Guidelines for Prevention and Treatment of Hypertension (Revised Edition, 2018). Chin J Cardiovasc Med, 2019, 24(1): 24-56.

6 Tang HY, Zhu JC, He HY, et al. The preparation and reliability and validity of the Hypertension Treatment Compliance Scale. J Third Mil Med Univ, 2011, 33(13): 1400-1403.

7 Tang HY, Li M, Jiang M, et al. Development of the attitude and belief evaluation scale for hypertension treatment and evaluation of its reliability and validity. Chin J Hypertens, 2011, 19(9): 861-866.

8 Clark DB, Martin CS, Chung T, et al. Screening for underage drinking and diagnostic and statistical manual of mental disorders, 5th Edition, alcohol use disorder in rural primary care practice. J Pediatr, 2016, 173: 214-220.

9 Ware JE. SF-36 health survey manual and interpretation guide. Lincoln, RI: Quality Metric,, 2000.

10 Kitaoka M. The relationship between hypertension and health-related quality of life: adjusted by chronic pain, chronic diseases, and life habits in the general middle-aged population in Japan. Environ Health Prev Med, 2016, 21(4): 215-223.

$11 \mathrm{Xu}$ XL, Rao YS, Shi ZM, et al. Hypertension Impact on Health-Related Quality of Life: A Cross-Sectional Survey among Middle-Aged Adults in Chongqing, China. Int J Hypertens, 2016, Article ID 7404957.

12 Zhi YP. Research progress on the quality of life of patients with hypertension. Cardiovasc Dis J Integr Trad Chin Western Med, 2018, 16(5): 562-566.

13 Li YN, Zhang J, Xu SX. Analysis of the Factors Affecting the Treatment Compliance of 190 Elderly Patients with Hypertension in Rural Areas. Chin Nurs Manag, 2015, 15(3): 304-308.

14 He L. The effect of health education based on crosstheoretical model on compliance behavior of patients with hypertension. J Qilu Nurs, 2018, 24(8): 58-60.

15 Martha N, Hill RN. ASH Position Paper: Adherence and Persistence With Taking Medication to Control High Blood Pressure. J Clin Hypertens, 2010, 12(10): 
$11-17$

16 Zhong CJ. Investigation and analysis of treatment compliance of elderly hypertensive patients in a community in Changsha City. Med Inform, 2018, 31(14): 81-84.

17 Shi W, Zhou Q, Shen NG, et al. Relationship between treatment compliance, treatment attitude and belief and medical social support in elderly hypertensive patients in a district of Qiqihar City. Med Soc, 2019, 32(4): 90-94.

18 Zhu YR, Chen HF, Dimon. Relationship between selfhealth management ability and health literacy and social support in patients with essential hypertension. Chin Gen Pract Nurs, 2019, 17(14): 1685-1688. 